\title{
APROXIMACIÓN A LA FIGURA DE JUAN DE OCHOA: ARQUITECTO E INGENIERO HIDRÁULICO ESPAÑOL DEL SIGLO XVI
}

\author{
AN APPROACH TO THE FIGURE OF JUAN DE OCHOA: \\ SPANISH ARCHITECT AND HYDRAULIC ENGINEER \\ FROM THE 16TH CENTURY
}

\section{JUAN LUQUE CARRILLO*}

\section{RESUMEN}

Juan de Ochoa Méndez (1554-1606) fue un arquitecto renacentista español que vivió en el tránsito del siglo XVI al XVII. Afincado en Córdoba, se trata de uno de los principales difusores del Manierismo arquitectónico en la geografía andaluza. Como su padre, Martín de Ochoa, fue un eficiente ingeniero, con una amplia labor en el campo de la hidráulica; y, en el ámbito de la arquitectura residencial, supo interpretar las necesidades de las élites cordobesas. No obstante, fue la arquitectura religiosa la que le concedió un particular reconocimiento, gracias a su cargo de maestro mayor de la catedral, el cual le permitió afrontar el cierre del proyecto quinientista para el templo, con la erección de la cúpula que preside el crucero y el cañón rebajado del coro.

Palabras clave: Juan de Ochoa, siglo XVI, Córdoba, cantería, catedral.

\section{ABSTRACT}

Juan de Ochoa Méndez (1554-1606) was a Spanish renaissance architect who lived from the second half of the 16th century to the beginning of the 17th. Based in Cordoba, he is one of the major disseminators of architectural Mannerism in the peninsular area. Like his father, Martín de Ochoa, he was an efficient engineer with extensive work in the field of hydraulics; and in the field of residential architecture, he was able to interpret the needs of the Córdoba elites. However, it was religious architecture that granted him special recognition, thanks to his position as grand master of the Córdoba

* Doctorando en Historia del Arte, Universidad de Sevilla. Sevilla, España. Correo electrónico: juanluque317@gmail.com 
cathedral, which allowed him to face the closing of the temple project which the erection of the dome presiding transept and choir lowered canyon.

Keywords: Juan de Ochoa, 16th Century, Córdoba, stonework, cathedral.

Recibido: 30.09.14. Aceptado: 03.12.14.

$\mathrm{D}$

URANTE EL SIGLO XVI, como en otros muchos puntos de España, Córdoba mantuvo urbanísticamente la división de la ciudad heredada de la Edad Media: la Villa, vieja Medina, y la Ajerquía. Sin embargo fue un siglo de notables transformaciones urbanas, de grandes edificaciones oficiales, mejoras espaciales y de captación y difusión de los nuevos ideales renacentistas emanados de Italia, es decir un momento de vital importancia en que se llevó a cabo la transformación de la ciudad medieval en una ciudad moderna según los criterios y planteamientos del Humanismo.

Lógicamente es muy poco lo que queda en la actualidad de aquella Córdoba que en 1570 vio el monarca Felipe II (1556-1598), cuando los ciudadanos cordobeses gravitaban entre la zona comercial de la Feria y Calceteros y el centro noble en torno al Campo Santo de los Mártires. Para perpetuar esta visita real se levantó en las proximidades del Palacio Episcopal y la Aduana la Puerta del Puente, que pasó a ser la entrada principal a la ciudad. Construida en 1571 por el arquitecto Hernán Ruiz III (1534-1606), su aspecto de arco triunfal adintelado pone de manifiesto la importancia de la cultura renacentista en España (Villar, 1986). De hecho, su esquema manierista está basado en la portada de la Casa de los Páez de Castillejo, también en Córdoba, realizada a partir de un dibujo del boloñés Sebastiano Serlio (1475-1554).

Junto a esta arquitectura conmemorativa, plazas y fuentes públicas centran buena parte de la dedicación del urbanismo. Córdoba tuvo su plaza mayor en la Corredera, luego sustituida por la actual del siglo XVII, conservándose solo de su antigua urbanización el edificio de la Cárcel y las casas de doña Ana Jacinto de Angulo. En cuanto a las fuentes, elemento urbanístico de primer orden por su forma, belleza y uso, se conservan modelos de dos tipos: la fuente exenta, por un lado, cuyo mejor ejemplo se conserva en la popular Plaza del Potro, y la de pilón adosado, magníficamente ilustrada por la famosa Fuente del Rey, o de la Salud, de la localidad de Priego de Córdoba, construida en 1586 con diseño manierista bajo la dirección de Francisco del Castillo (1528-1586). 
Afortunadamente, de la abundante nómina de arquitectos y maestros canteros activos en Córdoba en los siglos XVI y XVII, la investigación va destacando cada vez más la figura de Juan de Ochoa. Sobre este maestro había un cierto número de referencias documentales, casi todas extraídas de los protocolos cordobeses por Rafael Ramírez de Arellano, además de otros datos proporcionados por Alberto Villar. En 1970 José Valverde Madrid publicó un primer estudio de conjunto sobre el arquitecto, ofreciendo un bosquejo biográfico y, sobre todo, realizando la primera recopilación de sus obras, aportando en algunos casos interesantes materiales gráficos. Con el presente trabajo, fruto de la revisión documental que llevamos a cabo en el Archivo Histórico-Provincial de Córdoba, se pretende aportar una nueva visión sobre este maestro cantero, concerniente tanto a aspectos biográficos como a su actividad profesional en la ciudad de Córdoba, permitiéndonos ampliar el catálogo de sus obras, en unos casos conservadas y en otros solamente documentadas.

\section{ASPECTOS BIOGRÁFICOS}

Uno de los aspectos más interesantes es el de conocer cuándo y dónde nació el arquitecto. En este sentido, José Valverde (1970) publicó, aunque con errores de transcripción, su partida bautismal, que dice así: "En 27 dias del mes de dizienbre año de 1554 años batizo Andres Martinez Batista a Ju. hijo de Martin de Ochoa y de Ana Mendez su mujer y fuero compadres Melchior de Pineda y Xpoval de Mesa racioneros comadres Maria de Valençuela y Fca. De Valençuela. Andres Martz. Baptista”. Sus padres, el maestro de cantería Martín de Ochoa y Ana Méndez, estarían probablemente avecindados en los aledaños de la catedral, cerca de la parroquia del Sagrario, donde el arquitecto fue bautizado. En cuanto a su domicilio, estuvo ubicado en la collación de Santa María, de origen medieval y configurada en tiempos del rey Fernando III “el Santo" tras la Reconquista de 1236. No es fácil delimitar con precisión el lugar concreto en que vivió, ni si el arquitecto cambió de vivienda al ingresar en el cuerpo de alarifes, pues hasta el momento no se han hallado arrendamientos de casas firmados por Ochoa. Como suele ser habitual, la documentación alude de manera genérica a la collación, sin especificar la calle concreta en la que estaba la casa. No obstante, una escritura extendida en junio de 1571 presenta por vez primera a Juan de Ochoa viviendo en la collación de Omnium Sanctorum, donde permaneció junto 
a su esposa, hijos y madre hasta 1606 en que firmó su último contrato de obra para la iglesia mayor de la localidad cordobesa de Santaella.

Hasta ahora, en lo que a la actividad profesional se refiere, se tenía como su fecha más temprana 1577, año en el que acepta por aprendiz a Diego Coronado. Así mismo, se consideraba como su primera obra conocida la capilla mayor del monasterio de Santa Isabel de los Ángeles de Córdoba, proyecto en el que estuvo ocupado desde 1583 hasta 1587. Le seguiría algunos de los arreglos documentados de la casa de los Gome de Figueroa, marqueses de Villaseca, la actual Viana, y el claustro del antiguo cenobio agustino de Nuestra Señora de las Nieves, hoy patio principal del Círculo de la Amistad. Estas obras se han visto incrementadas no solo con las localizadas y estudiadas por Ramírez de Arellano en la capital, sino también con otras que hizo para algunos pueblos de la provincia, caso de Puente Genil, Priego de Córdoba, Luque o Hinojosa del Duque. Otros investigadores, por su parte, han dado a conocer que el maestro estuvo afincado en Sevilla en 1583 trabajando en la preparación del terreno seleccionado para erigir la Casa Lonja (Méndez, 1989), y finalmente, en 1606, en Arcos de la Frontera, Cádiz, terminando un puente que meses antes había proyectado Hernán Ruiz III (Ramírez de Arellano, 1903).

Por nuestra parte, gracias a la documentación manejada, podemos aportar nuevos datos que suponen un cambio sustancial con respecto a lo sabido hasta ahora. En primer lugar, podemos afirmar que la actividad de Juan de Ochoa debe adelantarse casi en una década. En efecto, las fuentes documentales han revelado que ya estaba activo en Córdoba en 1573, año en que es llamado junto a su padre y Fernando de Závala para reconstruir el puente que unía las localidades de Puente don Gonzalo y Mira Genil y que hoy, unidas, forman una sola: Puente Genil. La relación entre Ochoa y Hernán Ruiz III nos ha parecido de especial interés y es un aspecto que hasta el momento había pasado inadvertido. No sabemos qué unía a estos maestros, pero se pueden apuntar varias posibilidades; Hernán Ruiz, mayor que Ochoa 20 años, era ya un arquitecto consagrado, mientras que el joven maestro solo contaba con el respaldo y apellido paternal. Es bastante verosímil que se trate de una relación de discipulazgo, lo que explicaría algunas similitudes estéticas entre ambos, pero de momento no hay constancia documental. Otra posible causa de relación es la de tipo social, frecuente en los maestros cordobeses de la época; sin embargo no hemos podido establecer este tipo de vínculos entre ellos, si bien no lo descartamos. Sea cual fuera el origen de la relación, no se trató de un contacto esporádico, sino que se mantuvo durante el tiempo; así en agosto de 1583, ambos maestros 
se encontraban trabajando en Écija (Sevilla) en la conducción de las aguas a la ciudad y más adelante, en 1606, como se ha indicado, en Arcos de la Frontera terminando el puente que Hernán Ruiz había proyectado meses antes. Igualmente, en 1606, ambos maestros trabajaron conjuntamente en las obras de la catedral, Ochoa como maestro mayor y Hernán Ruiz a cargo de algunas obras de menor proyección que él mismo había solicitado al cabildo.

Todo ello permite suponer que sus comienzos deberían situarse en torno a 1573 o 1574, años en los que probablemente se examinara ante las autoridades gremiales, puesto que poco después aceptó a Diego Coronado como aprendiz.

Avanzada la centuria, en torno a 1590, el maestro casó con Francisca de Paula. Fruto de este matrimonio, en 1597, nació su única hija: Ana María. No obstante volvió a casar a finales del siglo al quedar viudo, con María de Clavijo, con la que tuvo al famoso misionero trinitario Luis de Ochoa (Valverde, 1970). Hija de don Alonso de Clavijo, en 1606 otorgó poder para vender unos censos heredados precisamente de su padre, figurando Ochoa al pie de la escritura como principal testigo, junto a su cuñado, también llamado Alonso de Clavijo, de profesión guadamecilero. Se desconoce cuánto tiempo duró el primer matrimonio, si tuvieron más hijos y cuándo se produjo la defunción de la esposa; es probable que muriera en torno a 15981600, pues como se ha indicado Ana María nació en 1597 y Luis, fruto del segundo matrimonio, en 1602.

Como se ha dicho más arriba, era conocido que Juan de Ochoa aceptó de aprendiz a Diego Coronado, considerado hasta el momento el primero y único, dado que no se conocen referencias que apunten a la existencia de otros discípulos. En efecto, en 1577 el arquitecto, que se dice vecino en Omnium Sanctorum, admite en su hogar a Coronado. Tal como se refleja en el documento, éste se incorporó como mozo aprendiz en octubre del referido año, entrando a formar parte de la familia Ochoa de Paula durante el tiempo que durara el periodo de aprendizaje. Además de las habituales condiciones relativas a la comida, casa, vestimenta y cama, comunes en este tipo de contratos, en este caso encontramos un dato curioso, que es la obligación que contrae Ochoa de regalarle a su aprendiz un sayal de paño negro, dos camisas de lienzo, un sombrero y unos zapatos en caso de superar el examen de oficial. Juan de Ochoa se declara en esta ocasión mayor de 23 años, mientras que el nuevo oficial dice tener solo un año menos.

Sobre Diego Coronado, llegado al hogar familiar el 7 de octubre de 1577, podemos añadir que era hijo de Diego Gutiérrez, natural de Sevilla y 
que se encontraba viviendo temporalmente en Córdoba, sin especificar la collación donde residía. A diferencia de otros contratos, en este se estipulan algunas condiciones novedosas, pues se hace constar que el aprendiz se obligaba a "seruir en su oficio y fuera del en todo lo que le mandare que sea onesto de façer y no se a de absentar de su poder y serviçio y si se absentare bolvera a el a servir dentro de quinze dias y si no lo cunpliere que pierda el tiempo que hubiere servido y vuelva a seruir de nueuo sin otra sentencia ni declaracion" (Archivo Histórico Provincial de Córdoba, España, 1577, octubre. Documento interno no publicado); en contrapartida el maestro se obligaba "de no poder echarle de su poder y serbicio sin causa alguna so pena de pagarle el tiempo que le ubiere seruido y lo que faltare por serbir" (Archivo Histórico Provincial de Córdoba, España, 1577, octubre. Documento interno no publicado). El período de aprendizaje se estipuló en dos años, desde el mismo día en que se extiende el texto de la escritura, y en caso de incumplimiento por cualquiera de las partes, se establecía una multa de 1.000 maravedíes.

No obstante, en los primeros años del siglo XVII aparece el arquitecto trabajando en la catedral junto al joven Blas de Masavel, que fue su gran colaborador en las obras del templo, además de su albacea testamentario, heredando a partir de 1606 el cargo de maestro mayor en el cabildo catedralicio. En este sentido, a la muerte del arquitecto, Masavel concluyó sus trabajos iniciados en la parroquia de Nuestra Señora de la Asunción de Santaella, centrados en el cuerpo de la iglesia, sacristía y baptisterio, pues Ochoa, desde 1600 hasta 1606, únicamente continuó la capilla mayor hasta cubrirla con la bóveda sobre pechinas, completando parte del proyecto trazado en 1559 por Hernán Ruiz II (Nieto, 1986).

Entre 1578 y 1599 el arquitecto aparece en varias ocasiones actuando en calidad de testigo, tanto en asuntos entre colegas como en causas particulares, lo que parece indicar un cierto grado de consideración social. Fuera del entorno de la arquitectura, Ochoa también gozó de estima y consideración entre otros artistas de la ciudad; así, en abril de 1590 contrajo la obligación de pagar, junto al pintor Pablo de Céspedes y el escultor Francisco Vera, 1.000 reales al señor Obregón, en concepto de 68 botones de oro, lo que a su vez parece indicar cierta solvencia económica (Valverde, 1970).

Una escritura extendida el 12 de julio de 1589 ofrece un dato de gran interés. En ella se menciona por primera vez a Juan de Ochoa "maestro mayor de las obras de Cordoua", lo cual hace suponer que tal nombramiento debió efectuarse entre esta fecha y el día 5 del referido mes, en que se otorgó su anterior contrato de obra, apareciendo en él como "maestro de 
canteria". En el referido texto del 12 de julio de 1589 Ochoa contrató, junto al albañil Andrés Hernández Guadalupe, la construcción de unos molinos en la parada de Alharo, propiedad de don Bartolomé de Velasco (Archivo Histórico Provincial de Córdoba, España, 1589, julio. Documento interno no publicado).

La llegada en 1582 del obispo don Antonio Mauricio de Pazos y Figueroa a la diócesis cordobesa supuso un cambio de gran importancia en el desarrollo de las obras catedralicias. Desde la muerte en 1569 de Hernán Ruiz II, el cargo de maestro mayor de obras de la catedral lo había ostentado precisamente su hijo, Hernán Ruiz III. No obstante, con el obispo Pazos y Figueroa las relaciones entre Hernán Ruiz III y la autoridad diocesana entran en crisis; la personalidad atrevida y aventurera del arquitecto hubo de ser sin duda una de las causas fundamentales que protagonizaron el desencuentro, y tal llegó a ser la exasperación que en 1583 el prelado decidió destituirlo de su cargo, siendo en este momento cuando se nombró nuevo maestro mayor de obras a Juan de Ochoa. Desde este año, y hasta la fecha de su muerte en 1606, el arquitecto asumió la dirección de las obras de la catedral y obispado, trabajando también en algunos pueblos de la diócesis, tal es el caso de Pedroche, Hinojosa del Duque o Luque (Galera, 2011).

Sigue Ochoa trabajando sin cesar cuando, en febrero de 1599, se compromete a labrar la bóveda oval del crucero y el cañón rebajado del coro (Gómez, 1778), ajustándose a las condiciones trazadas por el obispo don Francisco de Reinoso y Baeza (1597-1601). En 1600 la obra estaba acabada y el arquitecto subcontrata con Francisco Gutiérrez Garrido, maestro natural de Antequera, la labor ornamental en yesos, conforme a traza y modelos que estaban hechos. Este proyecto, además de ser el más ambicioso y el de mayor extensión en el tiempo, posee además una interesante novedad: en el citado contrato de febrero de 1599, al margen del texto, Ochoa ilustra las condiciones de la escritura mediante dos dibujos a pluma de la traza de una pechina con todo su ornamento, de la cuarta parte de la bóveda oval, de su cornisa y de la traza de la linterna, tanto por dentro como por fuera, quedando visible la linterna desde el exterior (Nieto, 1995).

Las últimas referencias al maestro nos llevan hasta 1606, año en el que de nuevo, como ya se dijo, aparece relacionado con Hernán Ruiz III. Habiendo marchado este arquitecto en junio del referido año a Arcos de la Frontera para proyectar un puente que le había sido encargado, la muerte le sorprendió en julio del mismo año, a las pocas semanas de haberse iniciado las obras. El cabildo municipal llamó entonces a Juan de Ochoa para que continuara la construcción del viaducto. El arquitecto aceptó el encargo y 
se marchó sin permiso de la ciudad de Córdoba, puesto que en sesión de 25 de julio acordó el Ayuntamiento de Arcos "se escriba al duque mi señor suplicandole se sirva de escribir al corregidor de la ciudad de Cordoba y a la santa iglesia della para que den licencia a Juan de Ochoa maestro mayor para que venga a esta obra de la puente" (Ramírez de Arellano, 1903). Aun así, pudiendo documentar la presencia de Ochoa en esta obra, resulta difícil acotar su intervención. Sí se sabe que el puente quedó sin hacer, volviéndose a proyectar en 1620 y empezándose a construir de nuevo en 1650. A su vuelta de Arcos de la Frontera retomó las obras de la catedral cordobesa, no debiendo permanecer mucho tiempo al cargo de las mismas puesto que, según Valverde Madrid, redactó testamento el día 1 de octubre del referido año, falleciendo a los pocos días. Fallecido, su viuda presentó un memorial donde se enumeraban sus bienes, obras y deudas pendientes. Nuevamente aparece Blas de Masavel relacionado con el arquitecto, esta última vez figurando como su albacea testamentario (Valverde, 1970).

\section{OBRA RELIGIOSA}

El gran mecenas de primera hora para Ochoa fue don Luis Gome de Figueroa, Marqués de Villaseca, quien en 1583 le encargó su sepulcro en el monasterio de Santa Isabel de los Ángeles, por el que cobró el arquitecto 600 ducados. No obstante, el principal encargo que le hizo fue la capilla mayor de dicho monasterio, cuyo patronato ostentaba precisamente la familia en consideración a que sufragaron todos los gastos de la obra. Ochoa trabajó en este proyecto desde 1583 hasta 1587, levantando hasta la cornisa, completándose el resto en el siglo XVII con los arquitectos Sebastián Vidal y Bernabé Gómez del Río (Villar, 1986). Ochoa organizó un espacio sobre planta cuadrada, siendo el más cúbico de todo el manierismo cordobés, abierto por arco toral y decorado en el testero con un retablo arquitectónico. La portada de la iglesia, también encomendada a Ochoa, combina la sobriedad del marco, con pilastras jónicas empotradas en los flancos. Finalmente el frontispicio, curvo y partido en el centro, muestra una elevación central en semicírculo para alojar el relieve de la Visitación en un marco decorado con interesantes motivos geométricos.

Desde bien pronto las relaciones de Ochoa con el cabildo catedralicio fueron tan fructíferas como con el municipal; no olvidemos que el arquitecto había sido bautizado en la parroquia del Sagrario y que sus padrinos habían sido los canónigos Cristóbal de Mesa y Melchor de Pineda. Así, 
cuando en 1583 el obispo Pazos y Figueroa destituyó a Hernán Ruiz III no dudó en acudir a Ochoa para que ocupara el cargo de maestro mayor. Uno de los primeros trabajos del arquitecto como maestro de las obras del obispado fue la terminación de la torre de la parroquia de San Juan Bautista de la localidad de Hinojosa del Duque, campanario para el cual estuvo trabajando el maestro entre 1588 y 1590 y que recoge en sí las enseñanzas de Hernán Ruiz II, al resolverlo con tres cuerpos, el primero de planta rectangular para adaptarse a la base preexistente, el segundo cuadrado y el tercero octogonal (Galera, 2011). El cuerpo rectangular es un auténtico arco cuadrifronte, decorado en las fachadas mayores con serlianas de óculos cuadrados y pilastras toscanas con un dado de entablamento encima, recurso este muy característico de Ochoa. En las esquinas de los dos cuerpos inferiores, las pirámides embotadas con bolas acusan la influencia de Juan de Herrera y nuevamente de los Hernán Ruiz (Fig. 1). La impronta de este campanario es patente, en la misma comarca, en el de la parroquia de Belalcázar, del siglo XVII, inconcluso, diferente en su emplazamiento pero similar en la estructuración de la base rectangular.

Paralelamente, en 1588, aparece Ochoa trabajando en otra torre de iglesia parroquial ligada al nombre de Hernán Ruiz II. Era obispo de Córdoba don Francisco Pacheco (1587-1590) cuando se le encargó al arquitecto la terminación del campanario de la parroquia de El Salvador de la localidad de Pedroche (Gómez, 1778). La intervención de Ochoa se centró en la proyección del chapitel de planta circular, aunque se desconoce si es de traza propia o del maestro Ruiz. Sobre los tres cuerpos decrecientes, el arquitecto erigió el chapitel, con cierre cónico y rematado por una cruz de hierro labrada.

En la madrugada del 22 de septiembre de 1589 Córdoba fue testigo de una de las mayores y más trágicas tormentas acaecidas en la ciudad, seguida de un desastroso terremoto que dejó numerosos destrozos entre sus vecinos y principales monumentos. La fuerza del seísmo, como describe Ramírez de las Casas Deza, afectó a la catedral. En concreto el chapitel ochavado de madera que remataba el campanario se derrumbó, cayendo frente a una de las viviendas próximas a la Puerta del Perdón. Al respecto, Juan de Ochoa realizó las trazas del nuevo chapitel, cuyas condiciones se recogen en una escritura extendida en noviembre del referido año y encontrada en el protocolo del escribano Alonso Rodríguez de la Cruz. Se trata de un chapitel piramidal, cubierto de hoja de lata, con unas bolas de cobre en la cúspide sobre las que se colocó una veleta. Además de la cantidad acordada, el arquitecto recibió dos ducados por la redacción de las condiciones, firmando al pie del documento, junto a Ochoa, el carpintero Francisco de Herrera. 

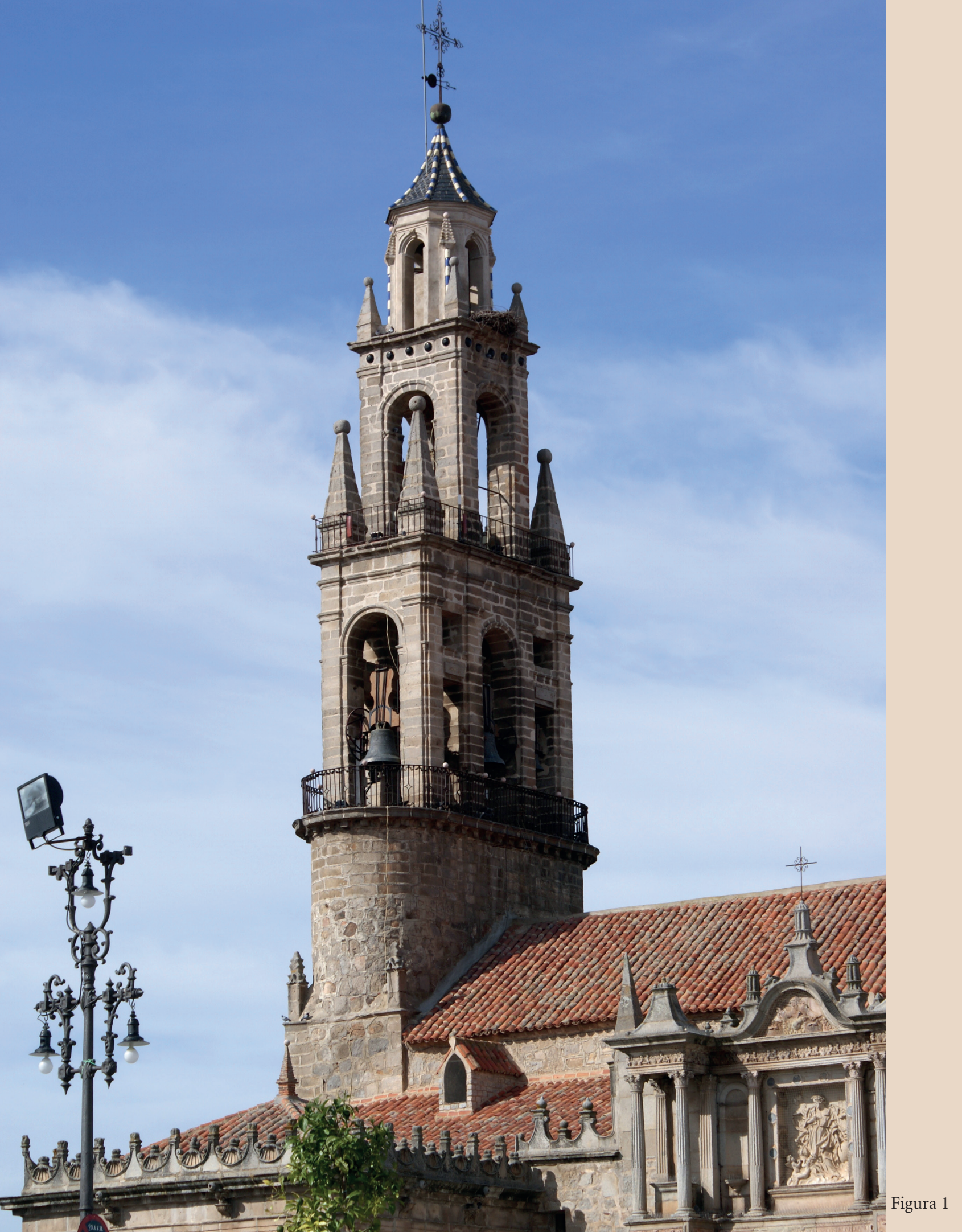
La linterna actual que corona el cuerpo de campanas es obra del arquitecto Gaspar de la Peña, pudiéndose apreciar sobre ella la escultura pétrea de San Rafael, obra de Bernabé Gómez del Río fechada en 1651 aproximadamente (Ramírez de las Casas, 1866).

Más adelante, en 1591, aparece documentado Ochoa en Luque, al sur de la provincia, delimitando las sepulturas de la nave central de la parroquia de Nuestra Señora de la Asunción. Únicamente en el texto de la visita pastoral de 1591 a la villa, el visitador hace referencia al arquitecto como maestro mayor de la obra pues nuevamente, en las cuentas del 1 de marzo de 1592, aparece Hernán Ruiz III dirigiendo las obras del templo (Estrada, 1993). La intervención de Ochoa en este proyecto no tuvo, por tanto, mayor trascendencia.

Una de las capillas mejor conservadas de la catedral de Córdoba es la de Santa Ana, adosada al muro este, en cuyo retablo central aparece un gran lienzo atribuido al pintor Pablo de Céspedes (1538-1608) con el tema de la Santa Ana Triplex con San Andrés y San Juan Bautista (Raya, 1998). Refundada en 1594 por los hermanos Andrés y Cristóbal de Mesa y Cortés, canónigos racioneros los dos, se trata de la capilla donde mejor puede apreciarse la influencia grecorromana de todo el templo mayor cordobés. En mayo de 1596 don Cristóbal de Mesa acudió a Ochoa, al que había apadrinado en su bautizo, para que cubriera el espacio, recibiendo por el trabajo $600 \mathrm{du}-$ cados. Para ello el arquitecto proyectó una bóveda sobre pechinas decorada con una interesante compartimentación en yesos donde recrea diferentes módulos y figuras geométricas (Ramírez de Arellano, 1903).

Con el obispo don Francisco de Reinoso y Baeza (1597-1601) las obras del templo mayor cordobés rozaron su fin pues, gracias a la dedicación y empeño personal del prelado, la nueva catedral de Córdoba quedó concluida (Gómez, 1778). A la hora de cerrar las bóvedas surgieron dudas sobre cómo hacerlo y sobre la posible resistencia de sus muros. Ello condujo al obispo a ponerse en contacto con el arquitecto Diego de Praves (15561620), maestro mayor de las obras de la catedral de Valladolid, para que opinara al respecto y propusiera posibles soluciones. El abovedamiento en ladrillo en lugar de cantería, más ligero y de menor peso, y los aletones que sirven de contrafuertes fue la solución planteada por el arquitecto vallisoletano. Esta visita de Praves a Córdoba debió efectuarse a finales de 1598-comienzos de 1599, pues el contrato con Juan de Ochoa por el cual el cabildo le concede la obra de la terminación del crucero y coro, no se firma hasta el 9 de febrero de 1599 (Nieto, 1995). Al año siguiente la obra estaba acabada y Ochoa subcontrata con Francisco Gutiérrez Garrido la labor decorativa en 
yesos. Toda la intervención del arquitecto en la catedral está perfectamente documentada. Dos contratos custodiados en el Archivo Histórico-Provincial de la ciudad, junto a la información extraída de las actas capitulares de la catedral, informan paso a paso de todo el proceso.

Para cubrir el crucero, el arquitecto diseñó una bóveda oval derivada en buena medida de la que Asencio de Maeda había trazado poco tiempo antes para la sala capitular de la catedral de Sevilla, pero con mejor decoración y dispositivo lumínico más efectista en el caso de Córdoba. Curiosamente, el citado Pablo de Céspedes había trabajado en el adorno de la obra sevillana y quizás también se tuviera en cuenta su opinión a la hora de decidir la forma ovalada, tan rara en el ámbito cordobés como frecuente en el manierismo italiano (Fig. 2). No obstante, la decisión de la planta recae de manera exclusiva en Praves, y su diseño en Ochoa. La bóveda monta sobre una gran cornisa con arquitrabes, frisos, frutas y follajes, motivos que después se volverían a repetir magníficamente en la gran cornisa del hastial de los pies del coro. Seguidamente el arquitecto trazó para el coro una bóveda de cañón rebajada, decorándola según el modelo y distribución que Miguel Ángel dio al techo de la Capilla Sixtina, en Roma, a principios del siglo XVI. Aunque varíen concepto ornamental y programa iconográfico, el parecido es evidente. En la Capilla Sixtina todo gira en torno a la representación de las escenas del Génesis, mientras que en el caso cordobés aparecen los escudos de los obispos que intervinieron en su construcción, ángeles, santos y la patrona y titular del templo: Santa María de la Asunción.

Finalmente, la última obra religiosa del arquitecto documentada hasta el momento está relacionada con la capilla mayor del templo de la Asunción de Santaella. En ella, entre 1604 y 1606 Ochoa terminó de levantar sus muros, desde donde lo había dejado Hernán Ruiz III, y cubrió el espacio mediante una bóveda sobre pechinas. El arquitecto cobró por ello 2.000 ducados, incluyendo en el precio el equipo de trabajo que llevó a cabo el encargo, compuesto por tres maestros y diez peones. El cuerpo de la iglesia, sacristía y baptisterio fueron terminados a mediados del siglo XVII, bajo la dirección de Blas de Masavel, respetando las trazas diseñadas en 1559 por Hernán Ruiz II (Nieto, 1986).

\section{TRABAJOS CIVILES}

Las primeras noticias documentales sobre la intervención de Juan de Ochoa en obras civiles de cierta envergadura datan de 1583. Fuera de su Córdoba 
natal, en Sevilla, se encuentra trabajando en la preparación del terreno seleccionado para erigir la Casa Lonja, espacio hasta entonces ocupado por las Herrerías del Rey y la Casa de la Moneda. En marzo del referido año se inició la operación de derribo de las edificaciones y de varias viviendas colindantes que fueron adquiridas como consecuencia de la insuficiencia del solar. Estos trabajos de demolición fueron dirigidos por Ochoa hasta diciembre de 1583, en que fue sustituido en su cargo por el arquitecto Juan de Minjares, recién llegado de las obras del monasterio de San Lorenzo de El Escorial, Madrid (Morales, 1995).

No obstante, años atrás, ya encontramos activo al arquitecto en Córdoba trabajando en obras de conducción de aguas. Así aparece documentado en junio de 1571, reedificando un pozo en la huerta de Cercadilla, propiedad de don Pedro de Valencia, por 340 ducados.

Nuevamente relacionado con trabajos de canalización de agua aparece Ochoa en 1573, en concreto reedificando el puente que unía los pueblos de Puente don Gonzalo y Miragenil. En abril del referido año, el padre del arquitecto, Martín de Ochoa, y Fernando de Zavala otorgaron licencia a Juan de Ochoa para sacar y llevar al citado puente la piedra, dovelaje y sillería necesaria para su posterior reedificación. Este viaducto fue trazado en 1561 por Hernán Ruiz II y terminado en 1583 por Ochoa. No debiéndose por tanto las trazas del puente al arquitecto, se desconoce si a la muerte de Hernán Ruiz II Ochoa tomó el cargo de maestro de la obra, o si simplemente trabajó en su desarrollo supervisado por otro maestro. Todo parece indicar que debió asumir la dirección de la obra, aunque respetando el diseño del maestro Ruiz. Numerosas transformaciones en los siglos XVII y XVIII han alterado por completo el aspecto original del viaducto, conservándose únicamente de su arquitecto el pilar cruciforme central.

En esta misma línea vuelve a aparecer el maestro, en 1578, dirigiendo las obras de restauración y renovación del edificio de la Albolafia, en plena rivera del río Guadalquivir a su paso por Córdoba donde, hasta tiempos de la reina Isabel la Católica, estuvo funcionando una máquina de elevación de agua, construida por los musulmanes en el siglo IX y que precisamente ellos denominaron Albolafia, quedándose finalmente la construcción con este nombre. Posteriormente, en 1492, la reina Católica, alojada en el Alcázar, mandó desmontar la rueda de la noria al resultarle muy molestosa. Así, desde 1492 y hasta 1578, la construcción estuvo desprovista de noria, siendo en este año cuando, por petición de las monjas del convento de Jesús María, Ochoa la restaurara, dándole de nuevo a la obra la utilidad con la que fue creada (Fig. 3). Desde entonces la noria fue reflejada en el sello del 


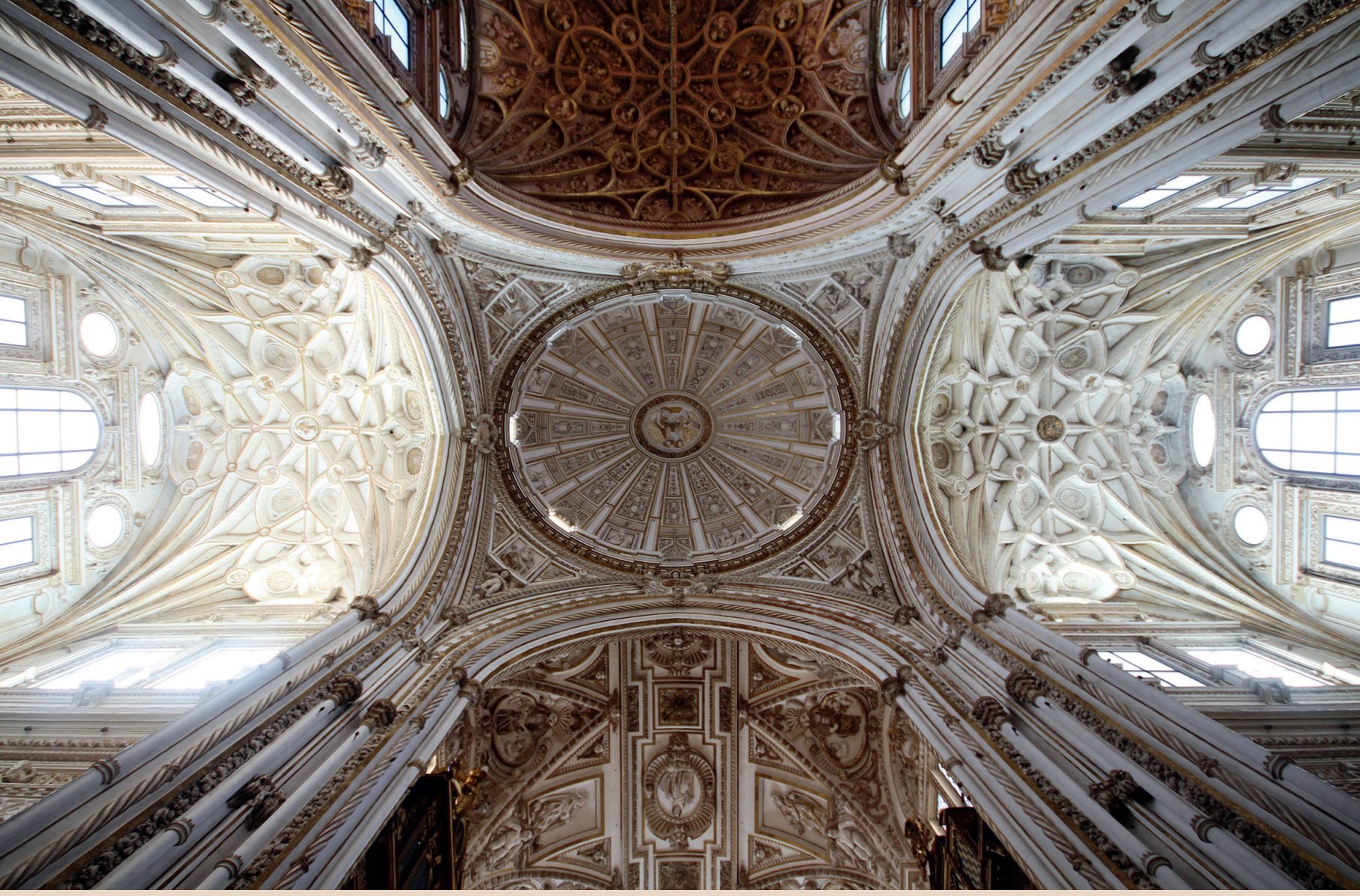

Figura 2

Figura 3

.

(3)

*

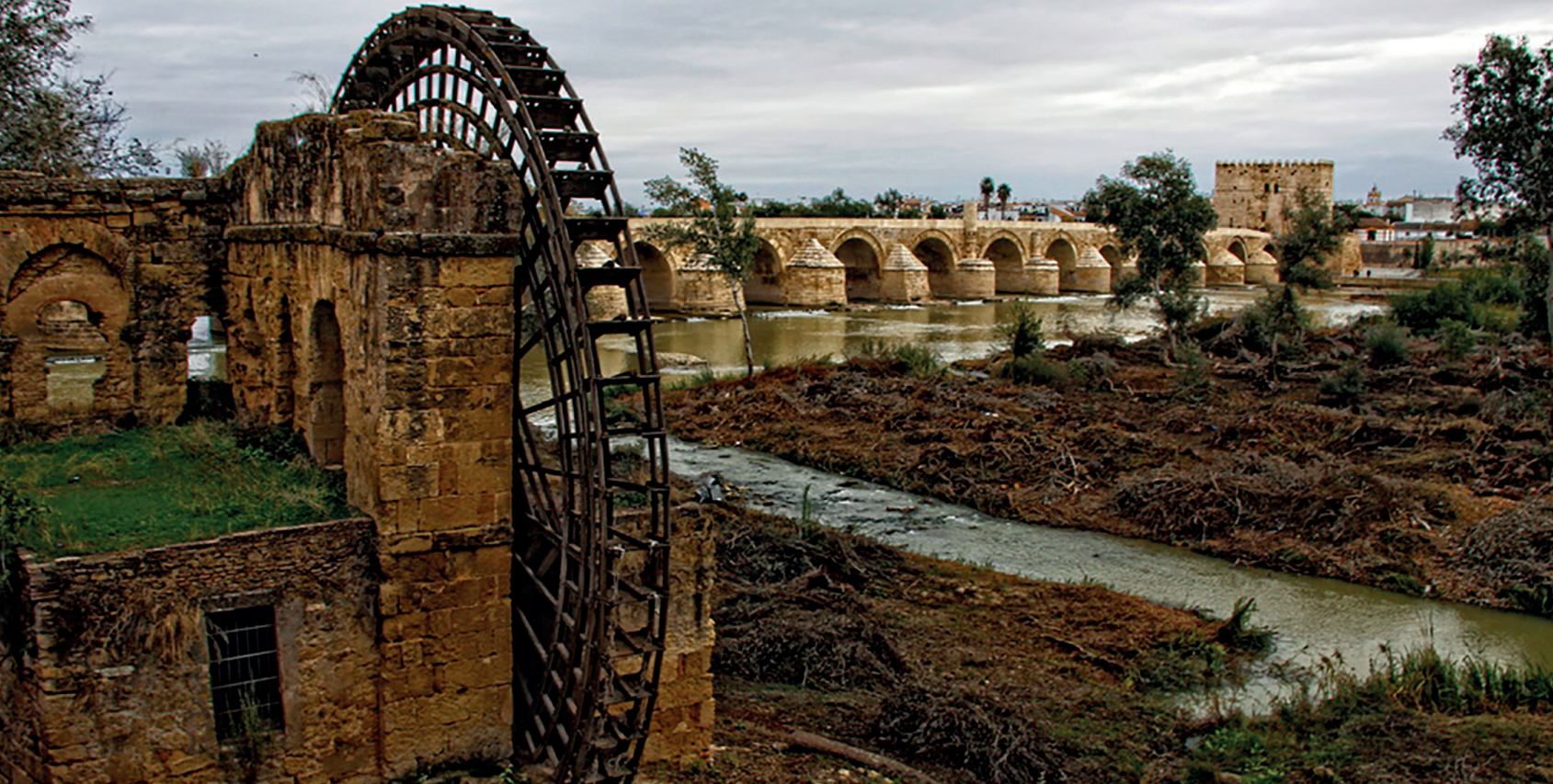


Concejo de la ciudad, junto a la catedral y el puente (Villar, Dabrio y Raya, 2005).

Más adelante, en 1583, Juan de Ochoa recibió 300 reales por acudir a Écija (Sevilla), donde presentó un proyecto de conducción de las aguas, arcas y fuentes de la ciudad, con el cual terminó el programa de abastecimiento de aguas diseñado en 1578 por Hernán Ruiz III. Este proyecto de Ochoa insistía en temas avanzados por sus predecesores, a la vez que incluía aspectos inéditos como la construcción de cuatro fuentes exentas de piedra, en otras tantas plazas ecijanas. No obstante, el proyecto del arquitecto no llegó a formalizarse pues, pregonadas las obras, Hernán Ruiz III fue encarcelado con el inicio de una larga causa judicial, nombrando finalmente el concejo ecijano a Francisco Fernández de Medellín nuevo maestro de la obra (Fernández y Morales, 1993). La intervención de Ochoa no tuvo pues mayor trascendencia.

Más importante y de mayor significación fue la intervención llevada a cabo entre 1584 y 1587 en el proyecto urbanístico cometido en la cordobesa Plaza de la Corredera, cuya labor del arquitecto se centró en la proyección del edificio de la Cárcel y Casa del Corregidor. En esta obra Ochoa reflejó una arquitectura plenamente manierista, especialmente en la fachada, austera, sobriamente decorada y compartimentada por las líneas verticales que constituyen las pilastras del primer y segundo cuerpo en correspondencia, y por las horizontales del friso de separación de ambos cuerpos. Esta fachada debió estar enlucida, ocultando el lienzo de sillería y ladrillo, en contraste con el resto del conjunto de la Plaza, construido con tapial de cal, arena y tierra (Illescas, 1981). En su estructura original, el edificio debió constar de dos pisos, separados por un balcón corrido, con una balaustrada de piedra que lo remataba, desde donde los más ilustres personajes de la sociedad cordobesa del momento asistían a los principales actos lúdicos. Interiormente, todo el espacio quedaba organizado en torno a un patio rectangular, con fuente en el centro y doble galería porticada a base de arcos de medio punto sobre columnas toscanas, quedando todas las dependencias perfectamente dispuestas y organizadas: calabozos, salas de audiencias, aposentos del alcalde, de los nobles y capilla.

Lejos de la perfección clásica de la arquitectura italiana de comienzos del Quinientos, la obra de Juan de Ochoa muestra un notable interés por la experimentación. Es una obra novedosa, interpretada de manera personal, consistente en la formulación y desarrollo de una serie de principios cuya relación con los códigos clásicos se rompe, llegando a desaparecer por completo. Es una arquitectura fundamentalmente dual, donde la doble función 
(práctica y decorativa) se da con frecuencia. En este sentido, en el terreno de la arquitectura residencial, Ochoa supo cubrir las necesidades de las principales élites cordobesas del momento, teniendo al respecto varios ejemplos documentados de viviendas particulares. De todos ellos cabe mencionar las casas que en 1577 proyectó para doña Beatriz de Monsalve, para las cuales necesitó ocho dinteles de piedra que extrajo de la cantera de Pedrera por el precio de 4 reales cada uno. Al año siguiente también construyó la casa de los señores Francisco Murillo y Ruy Pérez de Murillo, maestrescuela el primero y chantre de la catedral de Córdoba el otro. En el texto de la escritura se mencionan doce mármoles con sus aparejos de basas y capiteles de ocho cuartas y media de largo y una tercia de grueso, y las basas y capiteles de una cuarta de alto. De ambos ejemplos solo se conserva la documentación, desconociéndose su emplazamiento y las características que debieron mostrar (Ramírez de Arellano, 1903).

Los frecuentes arreglos documentados de la Casa de los Gome de Figueroa permiten atribuir a Ochoa la obra en piedra de su portada, de 1590 aproximadamente, en la que se aprecian influencias tanto de Francisco del Castillo, en el diseño del pórtico, como de Hernán Ruiz III, en las columnas empotradas que flanquean la arquitectura del balcón (Villar, Dabrio y Raya, 2005). Su originalidad radica, sin embargo, en los planteamientos estructurales, al disponer la portada en línea con un ángulo del patio, encajada entre dos crujías convergentes sobre el plano de la entrada. El segundo cuerpo muestra un balcón central, flanqueado por dos figuras pétreas de guerreros armados y acorazados. Finalmente sobre el balcón aparece un frontispicio, típico manierista, partido en la zona del vértice, donde descansa el escudo de los Villaseca.

Finalmente también va a ir unido el nombre de Juan de Ochoa a la historia del teatro español, por tratarse de uno de los más antiguos arquitectos de aquellos corrales de comedias que cobraron tanto protagonismo a mediados del siglo XVI y durante todo el Siglo de Oro Español. Era 1601 cuando el arquitecto se hallaba preparando los planos y diseñando las trazas del teatro erigido sobre el solar de la primitiva cárcel, en la antigua calle de las Comedias, actual Velázquez Bosco; un teatro de planta cuadrada, de más de 24 metros de lado, rodeado de murallones de un metro de grosor. Aunque desaparecido en la actualidad, se piensa que próximo a la fachada principal debió existir un primer cuerpo de 3,60 metros de profundidad, cuya planta baja albergaba un primer zaguán de uso general, dos cuartos para la venta de agua y adquisición de bancos, un vestuario, un vestíbulo de entrada para los comediantes, y otro zaguán reservado para los miembros 
del cabildo. La planta alta, por otro lado, albergaba un cuarto de tramoyas y dos dependencias más, de las cuales una, la de mayores proporciones, debió contener dos camarillas para vestuario de actores y guardarropía respectivamente. Por último, frente al vestuario bajo se alzaba el escenario, cubierto, de 5 metros de lado y 1,388 de altura con relación al nivel del patio sobre el que se proyectó, estando éste en su mayor parte descubierto, protegido únicamente por un toldo. Finalmente la obra fue entregada en junio de 1603. En su edificación el cabildo municipal invirtió 3.432 ducados, de los cuales 5.240 reales corresponden a la obra de albañilería, frente a los 9.788 que supuso las labores de carpintería. El valor del edificio, sin embargo, es muy superior a estas cantidades, ya que a ellas debe añadirse el precio del solar y el de los materiales de construcción procedentes del derribo de los calabozos de la antigua cárcel (García, 1990).

Por último, a modo de conclusión, queremos referirnos a la figura de Juan de Ochoa como una de las personalidades más destacadas e indispensables del Renacimiento cordobés, un arquitecto con una amplia labor en el ámbito de la arquitectura religiosa, civil y también en el campo de la hidráulica, que supo interpretar las exigencias impuestas por las dos grandes empresas constructivas del momento: el concejo municipal y el cabildo catedralicio. No obstante a pesar de todo lo dicho, la figura de Ochoa quedó eclipsada por la dinastía de los Hernán Ruiz, cuyos tres miembros ocuparon una posición dominante en la arquitectura cordobesa desde finales del siglo XV hasta principios del XVII. Este hecho queda justificado por la relativa falta de atención ofrecida por la historiografía tradicional al arquitecto. Confiemos en que la revisión documental nos ayude a seguir ampliando su catálogo y a situar su labor profesional en el lugar que justamente le corresponde dentro de la arquitectura cordobesa del último tercio del Quinientos.

\section{REFERENCIAS}

Estrada, V. (1993). La iglesia parroquial de Luque (1586-1992). Córdoba, España: Excma. Diputación Provincial de Córdoba.

Fernández, M. y Morales, A. (1993). Hernán Ruiz II y el abastecimiento de aguas a Écija. En AA. VV., Actas del III Congreso de Historia de Écija en la Edad Media y Renacimiento (pp. 455-468). Écija, Sevilla: Fundación el Monte.

Galera, P. (2011). Arquitectos y maestros canteros en Andalucía en el Renacimiento. En J. Rubio (coord.), Artistas andaluces y artifices del arte andaluz, tomo 37 (pp. 230-274). Sevilla: Publicaciones Comunitarias. 
García, A. M. (1990). Casa de las comedias de Córdoba (1602-1694): contribución a su historia documental. Criticón, 50, 23-40.

Gómez, J. (1778). Catálogo de los obispos de Córdoba y breve noticia histórica de su iglesia catedral y obispado, tomo II. Córdoba, España: Oficina de don Juan Rodríguez.

Illescas, M. (1981). Evolución urbanística de la Plaza de la Corredera. Axerquía, $5,159-176$.

Méndez, C. (1989). La Casa Lonja de Sevilla. Aparejadores, 4, 11-15.

Morales, A. (1995). Lonja de mercaderes y Archivo General de Indias. En P. González (coord.), Archivo General de Indias (pp. 64-91). Barcelona: Lunwerg.

Nieto, M. (1986). La iglesia parroquial de Santaella. En J. Aranda (coord.), Santaella: estudios históricos de una villa cordobesa (pp. 161-196). Santaella, España: Círculo de Labradores.

Nieto, M. (1995). La Catedral de Córdoba. Córdoba, España: Publicaciones del Monte de Piedad y Caja de Ahorros de Córdoba.

Ramírez de Arellano, R. (1903). "Artistas exhumados". En AA.VV., Boletín de la sociedad española de excursiones, 124, 135-140.

Ramírez de las Casas, L. M. (1866). Descripción de la Iglesia Catedral de Córdoba. $4^{\text {a }}$ edic., Córdoba, España: Imprenta de Rafael Rojo y compañía.

Raya, Ma. A. (1998). Catálogo de las pinturas de la catedral de Córdoba. Córdoba, España: Publicaciones del Monte de Piedad y Caja de Ahorros de Córdoba.

Valverde, J. (1970). "Juan de Ochoa, el arquitecto de la catedral cordobesa". Omeya $14, \mathrm{~s} / \mathrm{f}$.

Villar, A. (1986). “La arquitectura del Quinientos”. En M. Guarinos (dir.), Córdoba y su provincia Tomo III (pp. 209-233). Sevilla: Gever.

Villar, A., Dabrio, Ma T. y Raya, Mª A. (2005). Guía artística de Córdoba y su provincia. Córdoba, España: Fundación José Manuel Lara. 